\title{
Plan de negocio para la creación de una Mipymes para elaboración de mascarillas faciales caseras.
}

\section{Business Plan for the creation of a development of Mipymes for homemade facial masks.}

Velasteguí López Efraín. ${ }^{1}$, Castro Romero Anggie. ${ }^{2}$

\section{DOI: https://doi.org/10.33262/visionariodigital.v1i1.235}

\section{Resumen.}

Será una empresa que se dedique a la elaboración y comercialización de mascarilla 100 por ciento naturales para el rostro, hecho a mano con materiales naturales tanto en los ingredientes utilizados, así como en los envases y etiquetas del producto que son totalmente reciclables. El análisis realizado a este indica que presenta varias oportunidades, las mismas que puede ser aprovechada para que este plan tenga un efectivo desenvolvimiento. Se ha confirmado que algunos clientes tienen atracción y cambios de hábitos de consumo de las personas hacia productos preferencias. La microempresa contara con cinco empleados, cuyas actividades estarán estratégicamente definidas para asegurar la eficiencia en la producción de los productos.

Desarrollare las siguientes mascarillas faciales con ingredientes naturales. Para limpiar: avena y yogurt, Para limpiar a profundidad: exfoliante de azúcar, Para desinflamar: café y cocoa, Para relajar: plátano, Para desinfectar: leche de magnesia, Para desinflamar y desinfectar: cebolla y avena, Para relajar: arcilla, Para limpiar y refrescar: pepino, Para regenerar: exfoliante de miel, menta y limón, Para disminuir las arrugas: zanahoria y yogurt, Para hidratar profundamente: aceite de oliva extra virgen, Para exfoliar y rejuvenecer: exfoliante de almendra y miel, Para desinfectar y refrescar: rosas, Para hidratar: aguacate, Para desinflamar y desinfectar: aspirina, Para suavizar e iluminar: papaya, Pará desaparecer los granitos: cilantro, Pará relajar: lavanda, Para retrasar los signos de la edad: miel de maple. Todos y cada uno de estos ingredientes serán utilizados para la limpieza facial en damas y caballeros.

Palabras clave: Planeación empresarial, Investigación de mercado, Mascarillas naturales.

\footnotetext{
${ }^{1}$ Universidad Técnica de Ambato, Ambato, Ecuador, le.velastegui@uta.edu.ec ${ }^{2}$ Universidad Técnica de Cotopaxi Extensión la Mana.Ecuador,anggie-cg@ hotmail.com 


\begin{abstract}
.
It will be a company that dedicates itself to the elaboration and commercialization of 100 percent natural mask for the face, made by hand with natural materials both in the ingredients used as well as in the containers and labels of the product that are totally recyclable. The analysis made to this indicates that it presents several opportunities, the same ones that can be exploited so that this plan has an effective development. It has been confirmed that some clients have attraction and changes in consumer habits of people towards products preferences. The microenterprise will have five employees, whose activities will be strategically defined to ensure efficiency in the production of the products. I will develop the following facial masks with natural ingredients To clean: oatmeal and yogurt, To clean at depth: sugar scrub, To deflate: coffe and cocoa, To relax: banana, To disinfect: milk of magnesia, To desinflamar and disinfector: onion ang oats, To relax: clay, To clean and refresh: cucumber, To regenerate: exfoliating honey, mint and lemon, To reduce wrinkless: carrot and yogurt, To deeply hydrate: extra virgin olive oil, To exfoliate and rejuvenate: almond and hobey scrub, To disinfect and refresh: roses, To moisturize: avocado, To reduce inflammation and disinfect: aspirin, To soften and lighten: papaya, To disappear granites: coriander, To relax: lavender, To delay the signs of age: maple syrup. Each and every one of these ingredients will be used for facial cleansing in ladies and gentlemen.
\end{abstract}

Keywords: Business planning, Market research, Natural masks.

\title{
I. Introducción
}

Según los actores (Barrow .2015.,Berry .2015., Bowman .2015.,Castillejo .2015.,Lloreda .2015 \& Meyer. 2015). Un plan de negocio no debe de ser estático si no dinámico y cambiante. Debemos revisarlo y adaptarlo a las necesidades y oportunidades del mercado, debe prepararse por todos los socios o promotores del proyecto por esta forma la implicación de todas las personas que van a intervenir en el análisis previo del mismo. Además contiene toda la información sobre la empresa y los objetivos que debe alcanzar, es decir, que en el plan de negocio debemos resumir como vamos a organizar nuestro trabajo y liderazgo empresarial, así como definir cómo se van a desarrollar las tareas necesarias para alcanzar el éxito marcado. El objetivo principal de un plan de negocios es la planificación, por eso es esencial se redacción antes de construir una empresa. Si bien es cierto que cada poco tiempo puede quedar desfasado dados los pasos y los resultados que vayamos obteniendo, esto no resta utilidad el plan de empresa, simplemente debemos tener en cuenta que habrá que ir actualizando y renovando el plan de negocio redactado inicialmente.

"Un plan de negocio se debe especificar principalmente en realizar un estudio de mercado para ver si lo pensado será factible dentro del desarrollo mercantil y a la toma de decisiones necesarias de cada uno de los ámbitos necesarios para la creación de empresas. Entre ellos encontramos diferentes estrategias sobre el proceso productivo, el 
personal, el marketing, etc. Un plan de negocio es un documento escrito de unas 30 cuartillas que incluyen básicamente los objetivos de tu empresa, las estrategias para conseguirlos, la estructura organizacional, el monto de inversión que requieres para financiar tu proyecto y soluciones para resolver problemas futuros (tanto internos como del entorno).Si bien cada proyecto es diferente, todo parte debe tener una idea y materializarla por escrito con base en cinco puntos básicos:

-Estructura ideológica. Incluye el nombre de la empresa, así como la misión, visión, valores y una descripción de las ventajas competitivas del negocio.

Figura: 1 Estructura ideológica

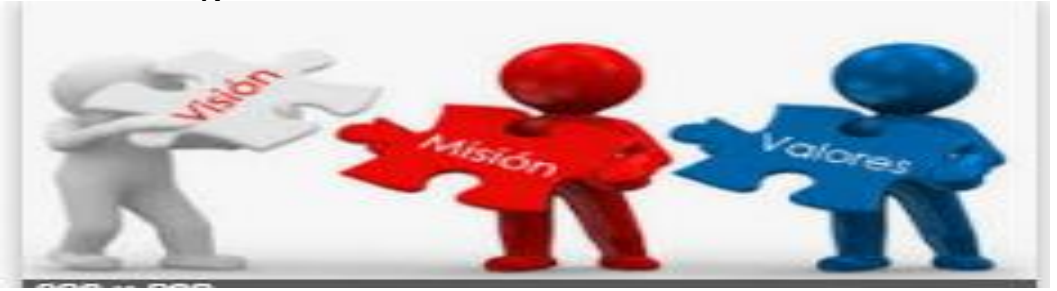

Autor:pizzería el chefitaliano

Fuente:planDenegoscios@gmail.com

-Estructura del entorno. Se fundamenta en un análisis de las fortalezas y debilidades de la empresa, así como del comportamiento del sector.

Figura: 2 Estructura del entorno

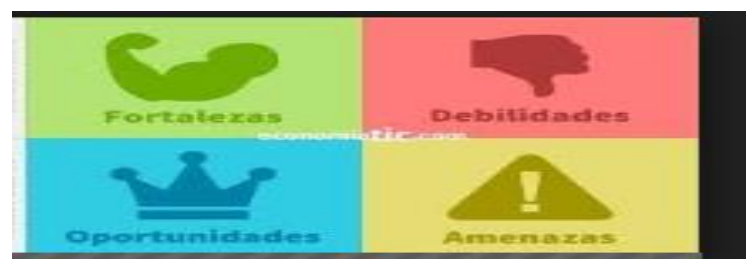

Autor: Johana Rodríguez

Fuente: wordpress.com

-Estructura mecánica. Aquí se enlistan las estructuras de distribución, ventas, mercadotecnia y publicidad, es decir, que acciones hay que ejecutar para lograr el éxito de la idea de negocios.

Figura: 3 Estructura mecánica

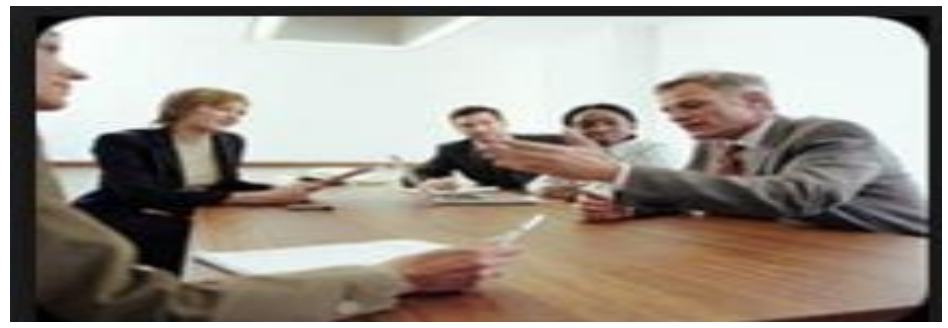

Autor: Geff Endara

Fuente: http://mktwth.blogspot.com 
-Estructura financiera. Este punto es esencial pues pone a prueba-con base en cálculos y proyecciones de escenarios- la viabilidad de la idea hablando en términos económicos, y si genera un margen de utilidad atractiva.

Figura: 4 Estructura financiera

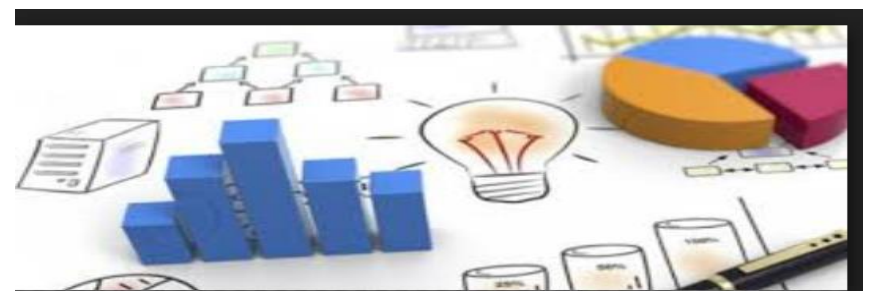

Autor: Empresa Financiera

Fuente: bancoestudiantil.com

-Recursos humanos. Ayuda a definir cada uno de los puestos de trabajo que se deben cubrir y determinara los derechos y obligaciones de cada uno de los miembros que integran la organización. No importa si estas arrancado tu solo, esto será tu base para asegurar el crecimiento de tu empresa.,"

Figura: 5 Recursos Humanos.

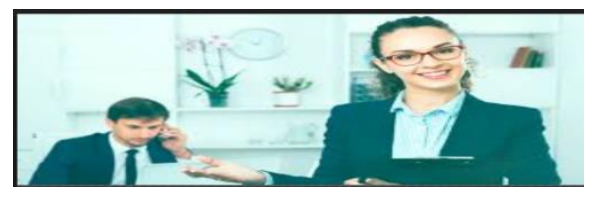

Autor: Alonzo de Molina

Fuente: www.esan.edu.pe

Según los actores (Garcia .2015.,Mook .2015.,Needham .2015., Prado .2015.,Smith .2015 \& Wilkins.2015). La Creación de una microempresa es actualmente uno de los mayores intereses, seguimiento, dedicación y esfuerzos tanto en los ámbitos académicos, políticos y sociales por su importancia demostrada en su capacidad para generar empleo, desarrollo y crecimiento económico en la sociedad actual. La capacidad de las microempresas es que se pueden hacer grandes empresas para adaptarse rápidamente a los cambios del entorno, así como su potencial innovación, convierte a estas empresas en un elemento clave en el desarrollo económico actual. A través de la figura y la visión del empresario (individual o grupal), existen múltiples factores que anteceden al procesos de creación y puesta en marcha de una nueva iniciativa empresarial y practica necesarias para que la probabilidad de que la iniciativa empresarial tenga éxito sea mayor. La creación de una microempresa necesita estructurar una serie de recursos materiales, humanos y económicos de cara a la consecución de una serie de objetivos establecidos por los emprendedores en la puesta de funcionamiento de su actividad. Para ello, se recurre generalmente al desarrollo de un plan de empresa. 
"Los micros empresas se conocen por la cantidad de personas que colaboran en la misma en donde se encargan de desarrollar actividades entre un equipo conformado por 5 personas o menos de ellas en un lugar pequeño pero no por decir que sea así va a obtener pequeños recursos. Ya es por ellos que hemos realizado esta investigación para saber todo acerca de las micro empresas por que como nuevos en ellos tendemos que saber. Entrepreneur" (que podríamos introducir como emprendimiento) y se comienza a definir quién es el empresario y que hace este, es decir qué papel juega en el proceso económico llamado función empresarial, que es crear y poner en marcha una empresa.

A pesar de buscarse el esencialismo metodológico, que pretende encontrar definiciones caracterizando el objetivo de estudio, prácticamente cada uno de los citados autores destaca una característica de lo que es la figura de la persona que emprende. Se dan muy diversas definiciones, algunas de las diferentes concepciones del empresario son:

- Empresario es aquel que asume el riesgo de la incertidumbre.

- Empresario es aquel que aporta capital.

- Empresario es un innovador.

- Es un decisor.

- Es el que consigue ser líder del sector.

- Es el manager o superintendente.

- Es un organizador y coordinador de recursos.

- Es un empleador de factores de producción.

- Es la persona que asigna recursos entre usos alternativos."

Figura 6. MiPyMES

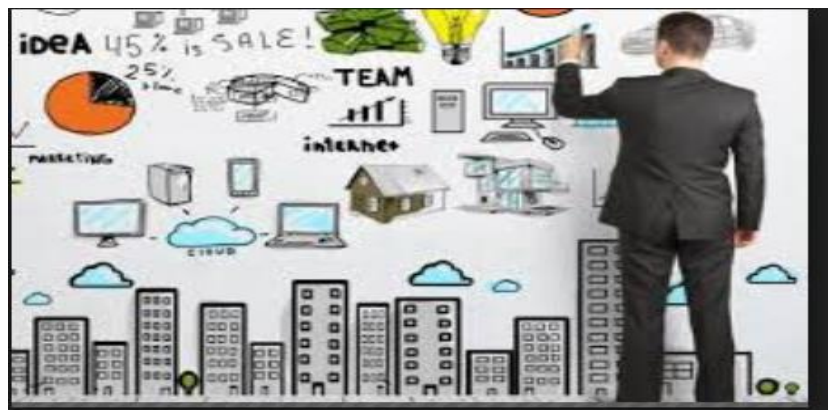

Autor: Mega informacion

Fuente: www.estudiosmega.net

Según los actores (Casey.2015.,Lamont.2015.,Mukherjee.2015.,Sanchez. 2015.,Tandon.2015 \& Yousem.2015). La Mascarilla faciales son un producto cosmético que se emplea de manera puntual para completar de forma sencilla el ritual de belleza diario. Para que los beneficios ostentan sean realmente efectivos hay que buscar la que más se adecue a cada tipo de piel y que contenga los activos necesarios que ayuden a mejorar la demis facial. Absorben la capa superior de la piel, Retener el agua en la piel e hidratarla, Atenuar las finas líneas de expresión, Exfoliar y retirar las células muertas de la superficie de la piel. Existen también mascarillas especializadas para contorno de ojos. También hay productos multifuncionales que pueden usarse como hidratante normal o como mascarilla hidratante de cualquier tipo de piel. Si se aplica una capa más gruesa y 
ISSN 2602-8506

Vol. 1 / No.1/ pág. 49-62. Enero-Marzo/2017

www.visionariodigital.org

se deja reposar durante algunos minutos. La mayoría de las mujeres olvidan la utilidad de este cosmético, que es un imprescindible en el cuidado del rostro ya que contiene múltiples propiedades para la demis.

"Las mascarillas faciales son un instrumento esencial para las mujeres ya que ayudan al cuidado de la piel facial a mantenerla sana y fuerte.

Los beneficios para cada piel vienen determinados por los ingredientes activos de la mascarilla facial. La experta establece que mascarilla habrá que utilizar dependiendo de las características del demis:

- Las pieles secas: deben utilizar una mascarilla hidratante.

Figura7.Las pieles secas

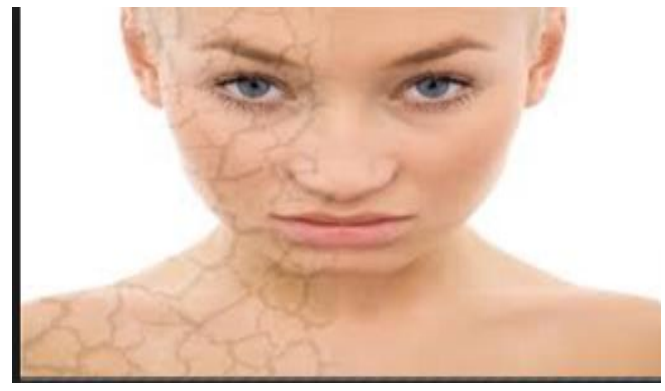

Autor: Barbará Gees

Fuente: barbaragees.com

- Las pieles acneicas: para ellas está indicada una mascarilla purificante y calmante.

Figura: 8 Las pieles acneicas.

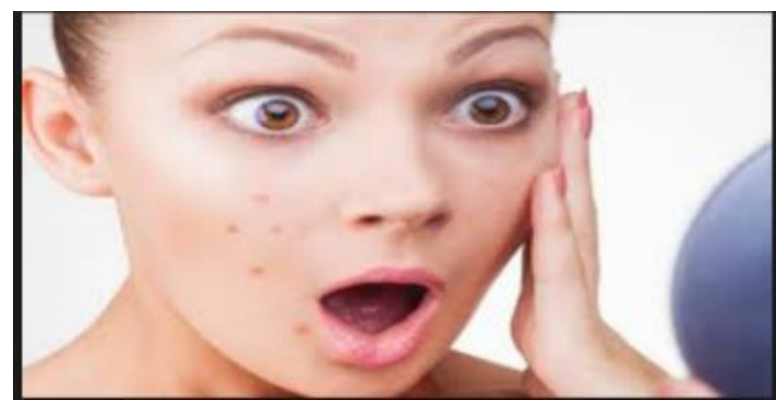

Autor: Saná Sana

Fuente: Sanasana.com

- Las pieles con rojeces: se benefician del uso de una mascarilla hidratante y Calmante. 
Figura 9. Las pieles con rojeces

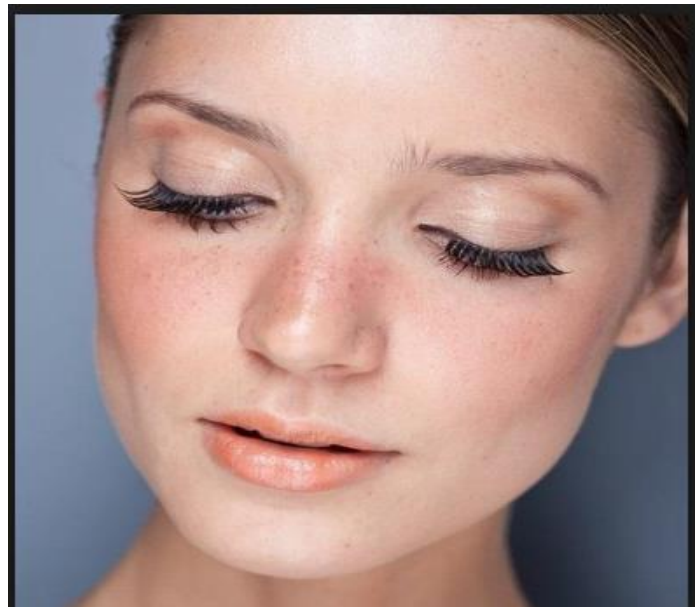

Autor: Link to media

Fuente: belleza.uncomo.com

- Las pieles fotoenvegecidas: han de emplear y una mascarilla con antioxidante que iluminen e hidraten la piel.

Figura 10. Las pieles fotoenvegecidas

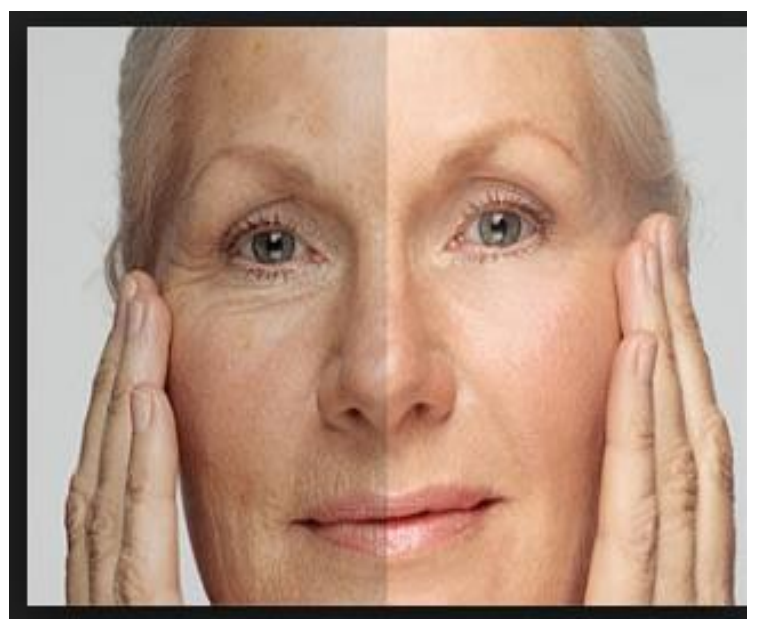

Autor: beauty and hope

Fuente: beautyandhope.es.tl

- Las pieles con manchas: necesitan activos que unifiquen el tono de la piel. 
Figura: 11 Las pieles con manchas

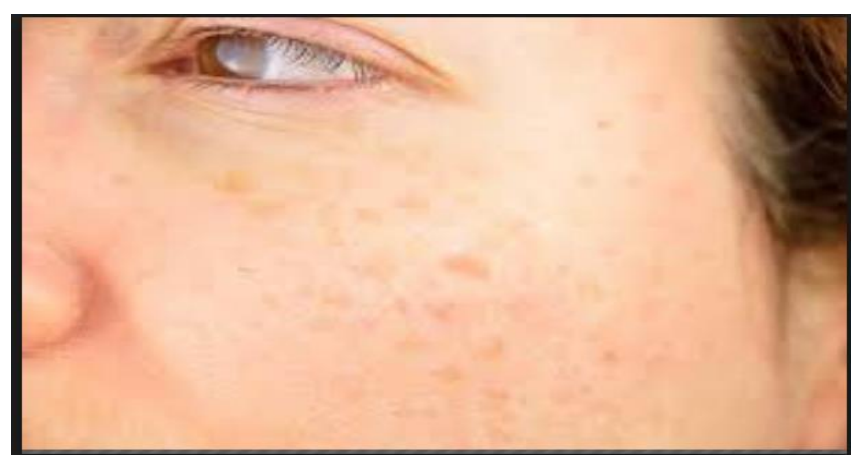

Autor: Carolina Betancourth

Fuente: mejorconsalud.com

\section{Diferentes texturas}

Este producto se puede encontrar en cremas y en sistema peel off, que se trasforma en una película fina transparente a medida que se va secando, lo que permite que se pueda retirar de una sola vez. También existe la mascarilla impregnadas en un tejido, también conocida como mascarillas tipo velo, que se adapta a la piel y ejerce una acción más oclusiva.

\section{Como aplica}

Ante de comenzar aplicar la mascarilla es recomendable tener las manos limpias y tonificadas. También, es posible exfoliar la pies, para que este mas respectiva a los activos que se apliquen posteriormente."

Figura: 12 Mascarillas Faciales

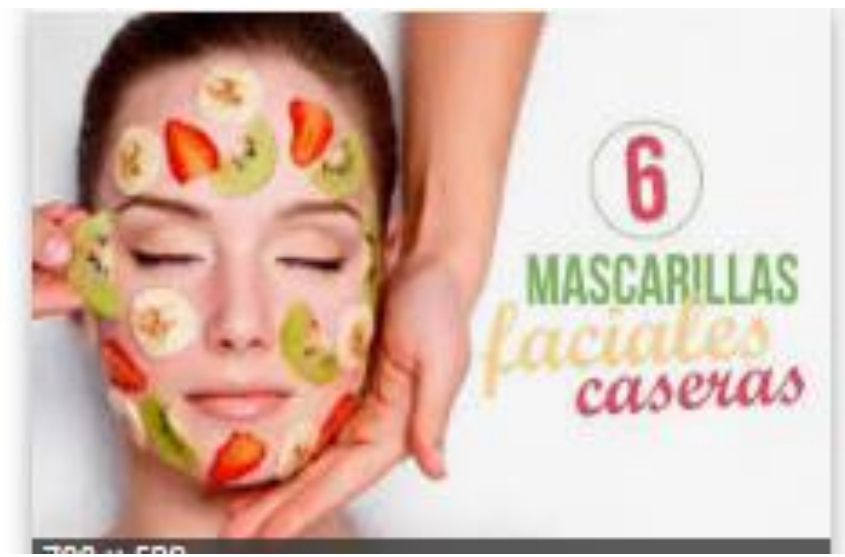

Autor: Valentina

Fuente: www.vix.com 


\section{Conclusiones.}

- Las mascarillas son una innovación que servirá para ayudar a remover la grasa de la cara esta hecho de ingredientes naturales.

- La preparación casera de las mascarillas, constituye un excelente truco de belleza, en especial para la limpieza y exfoliación del rostro.

- Estas recetas para hacer mascarillas faciales están fácil y rápida, que no podrás dejar de probarla.

- Las excelentes propiedades de los ingredientes para la limpieza de cutis, hacen del producto, uno de los favoritos, dentro de los recursos caseros para el cuidado del rostro facial.

- Es preferible para nuestro rostro utilizar mascarillas con ingredientes naturales y más si van a ser usados en nuestra piel facial.

- Cambiemos nuestros hábitos de comprar mascarillas inservibles que solo resequen la piel y dañan el medio ambiente, invierte en un producto que te dará beneficios y resultados rápidamente mediante su utilización diaria.

\section{Referencias.}

Guayllaguaman , D. (2015, 09 22). http://dorisaraceli.blogspot.com. Retrieved 0809 , 2017, from http://dorisaraceli.blogspot.com/2015/09/niveles-jerarquicos-de-unaempresa.html

Alvarez, R. (2016, Marzo 19). OPERACIONALIZACIÓN DE VARIABLES. METODOLOGIA DE LA INVESTIGACION. Retrieved from http://metinvc.blogspot.com/2012/02/t6a-variables-recurso-analitico.html

Barrow, C. (2015). The Business Plan Workbook: A Step-By-Step Guide to Creating and Developing... Kogan Page.com.

Bernal, C. (2010). Metodología de la investigación: administración, economía, humanidades y ciencias sociales. Colombia: Pearson.

Berry, C. (2015). Austerity Politics and UK Economic Policy. Springer.

Bonilla, E. (2015). La gestión de la calidad y su relación con los costos de desechos y desperdicios en las mypes de la confección textil. Universidad de Lima: Portal de revistas Ulima, 33, 1-14. Retrieved from Universidad de .

Bowman, A. (2015). What a Waste: Outsourcing and How it Goes Wrong. OXFORD.com.

Bravo, A. (2017, 04 02). timeofsoftware.com. Retrieved 08 09, 2017, from http://timeofsoftware.com/descubriendo-informacion/ 
Cagnazzo, L., Taticchi, P., \& Fuiano, F. (2010). Benefits, barriers and pitfalls coming from the ISO 9000 implementation: The impact on business performances. WSEAS Transactions on Business and Economics, 311-321.

Camejo, J. (2012, noviembre 28). Gestiopolis. Retrieved from Administración: http://www.gestiopolis.com/indicadores-de-gestion-que-son-y-por-que-usarlos/

Carazo, C. (2015, Agosto 21). El método de estudio de caso Estrategia metodológica de la investigación científica. Pensamiento y gestión, 1(20). Retrieved from http://rcientificas.uninorte.edu.co/index.php/pensamiento/article/viewFile/3576/ 2301

Carvajal, L. (2013, enero 18). Lizandro Carvajal. Retrieved from El método deductivo de investigación: lizandrocarvajal.com

Casey, E. (2015). Sex Differences in Sports Medicine. Springer Publishing Company.com.

Castillejo, L. E. (2015). Plan de negocios. Peru: Sextil Onlive, S.A.com.

Collazos, C. (2016, Junio 22). ALIMENTACIÓN Y NUTRICIÓN. Cien años de Cooperación al Perú, 1-13. Retrieved from http://www.paho.org/per/images/stories/PER/Documentos/cooperacion\%20peru/ PAG05.pdf

Correa, D., \& Orozco, J. (2015, Noviembre 22). Construcción de imagen de marca y reputación a través de campanas publicitarias de RSC. Redalic Sphera Pública(11), 273-289. Retrieved from http://www.redalyc.org/pdf/297/29729580016.pdf

Cuatrecasas, L. (2012). Organización de la producción y dirección de operaciones: Sistemas actuales de gestión eficiente y competitiva. Madrid: Díaz de Santos.

Díaz, P. (2014, Abril 22). Investigación cuantitativa y cualitativa . Unidad de Epidemiología Clínica y Bioestadística., 76-78. Retrieved from http://www.fisterra.com/mbe/investiga/cuanti_cuali/cuanti_cuali2.pdf

Escuela Europea de Excelencia. (2015). Nuevas Normas ISO. Retrieved from Adaptación a la nueva norma ISO 9001:2015: http://www.nueva-iso-9001-2015.com/

Fernández, R. (2010). La mejora de la productividad en la pequeña y mediana empresa. España: Club Universitario-ECU.

Frias, N. (2016, Enero 22). Alfa de Cronbach y consistencia interna de los ítems de un instrumento de medida. Estadistica moderna , 4. Retrieved from http://www.uv.es/ friasnav/AlfaCronbach.pdf

Fundación Telefónica. (2011). La sociedad de la informacion en España 2010. Barcelona: Ariel S.A.

GAD MUNICIPALIDAD DE AMBATO. (2016, Agosto 27). Acerca de nosotros: gadmatic.ambato.gob.ec. Retrieved from gadmatic.ambato.gob.ec: http://gadmatic.ambato.gob.ec/pcfiles/Proceso\%20Rendicion\%20de\%20Cuentas \%202014/11.\%20\%20CUMPLIMIENTO\%20DE\%20LA\%20EJECUCI\%C3\%9 3N\%20PRESUPUESTARIA/11.3.FUNCI\%C3\%93N\%20A)\%20\%E2\%80\%93 $\% 20($ HOJA\%2052).pdf 
Garcia, J. A. (2015). Comercializacion de productos y servicios en pequeños negocios. España: ic.Editorial.

García, O., Gonzalez, H., Rivera, L., \& Rivas, E. (2015, Febrero 19). SISTEMAS DE INFORMACION GERENCIAL. UNIVERSIDAD DON BOSCO, 2-33. Retrieved from https://s3.amazonaws.com/academia.edu.documents/36710601/PORTAFOLIO_ SIG.pdf?AWSAccessKeyId=AKIAIWOWYYGZ2Y53UL3A\&Expires=150464 4563\&Signature $=$ YpAdINrzjtRoSU\%2F8YPnGZKtnF\%2Bg\%3D\&responsecontent-disposition=inline\%3B\%20filename\%3DPORTAFOLIO_SIG.pdf

GestioPolis. (2013, junio 21). Retrieved from Gestiopolis.com: www. gestiopolis.com/sistema-de-gestion-de-la-calidad-segun-iso-9000/

Grajales, T. (2015, Marzo 08). TIPOS DE INVESTIGACION. Investipos. Retrieved from http://tgrajales.net/investipos.pdf

Hernandez, S., \& Palafox, G. (2012). Administración: Teoría, proceso, áreas funcionales y estrategias para la competitividad. México: McGraw Hill.

INEC. (2017, 08 09). ecuadorencifras.gob.ec. Retrieved from http://www.ecuadorencifras.gob.ec/documentos/webinec/Estadisticas_Sociales/TIC/2016/170125.Presentacion_Tics_2016.pd

Instituto Nacional de Estadística y Geografía. (2016, noviembre 8). Instituto Nacional de $\begin{array}{llll}\text { Estadistica } & y & \text { Geografía. Retrieved from INEGI: }\end{array}$ http://www.inegi.org.mx/default.aspx

Instituto Tecnológico de Sonora. (2014, Abril 1). Acerca de nosotros: biblioteca.itson.mx. Retrieved from biblioteca.itson.mx Web site: http://biblioteca.itson.mx/oa/dip_ago/introduccion_sistemas/p3.htm

ISO 9001. (2015). Requisitos para los Sistemas de Gestión de la Calidad. Ginebra: Secretaria Central de ISO.

Koontz, H., Weihrich, H., \& Cannice, M. (2012). Administración: Una perspectiva global y empresarial. México: McGraw-Hill/ Interamericana Editores S.A.

Lacalle, G. (2013). Gestión logística y comercial. Madrid: Editex, S. A. .

Lacalle, G. (2014). Operaciones administrativas de compraventa. Madrid: Editex, S. A. Lamont, R. (2015). Microbiologia e inmunologia oral. Manual Moderno.com.

Laundon, k. P. (2015). Sistemas de información gerencial: administración de la empresa digital. Madrid. Retrieved from https://books.google.es/books?hl=es\&lr=\&id=KD8ZZ66PF$\mathrm{gC} \&$ oi=fnd\&pg=PA210\&dq=que+son+los+sistemas+de+informaci\%C3\%B3n+ \&ots=hlhlubnZXD\&sig=Aur6Rw1G2rirqsJesfk4bqdN3vs\#v=onepage \&q=que\% 20son $\% 2010$ \% $\% 20$ sistemas $\% 20$ de $\% 20$ informaci\%C3\%B3n\&f=false

Llanusa, S., Rojo, N., Caraballoso, M., Roberto, C., \& Pérez, J. (2015, Mayo 19). Las tecnologías de información y comunicación y la gestión del conocimiento en el sector salud. Revista Cubana de Salud Pública, 31(3), 24-95. Retrieved from http://scielo.sld.cu/scielo.php?script=sci_arttext\&pid=S086434662005000300008

Lloreda, E. Z. (2015). Plan de Negocio. Madrid: OESIC.com. 
Marlats, M., Denegri, G. A., \& Lanfranco, J. (2015, Junio 29). Sistemas de informacion silvopastoriles: estimación de beneficios directos comparados con monoculturas en la pampa ondulada, Argentina. CATIE(8), 186. Retrieved from http://repositorio.bibliotecaorton.catie.ac.cr/handle/11554/6827

Martínez, R. (2014, octubre 15). SlideShare. Retrieved from Sesión 4indicadores producción: http://es.slideshare.net/Roxanamms/sesin-4-indicadores-produccion

Meyer, M. (2015). Venturing: Innovation ang Business Planning for Entrepreneurs. Lulu.com.

Ministerio de Coordinación de la Producción, Empleo y Competitividad. (2011). Agendas para la Transformacion Productiva Territorial Provincia de Tungurahua. Ambato.

Ministerio de industrias y de la productividad. (2016). Empresas textiles de Tungurahua. Ambato, Tungurahua, Ecuador.

Ministerio de Telecomunicaciones. (2016, Marzo 19). Plan Nacional de Telecomunicaciones y Tecnologias de la Información del Ecuador . 1-66. Retrieved from https://www.telecomunicaciones.gob.ec/wpcontent/uploads/2016/08/Plan-de-Telecomunicaciones-y-TI..pdf

Montaño, C., Cruz, A., \& Alcala, R. (2016, septiembre 28). Universidad de Colima. Retrieved from docentes.ucol: docentes.ucol.mx

Mook, L. (2015). Undertanding the Social Economy of the United States. UNIVERSITY OF TORONTO PRESS.com.

Mukherjee, S. (2015). Islam and the West: A Love Story? Paris: Publicado por Cambridge Scholars Publishing.com.

Namakforoosh, M. (2010). Metodología de la investigación. México D.F.: Editorial Limusa .

Needham. (2015). Micro-enterprise and personalisation:What size is good care? The POLICY PRESS.com.

Pérez, J. (2014, Mayo 08). CÓMO REDACTAR EL ANÁlisis DE LOS RESULTADOS. Asesoria. Retrieved from http://asesoriatesis1960.blogspot.com/2010/12/analisis-de-los-resultados.html

Plaza, D., Núquesi, A., \& Mejía, M. (2016, Abril 17). Implementación del sisytema de negociacion bursatilpara las pimes del Ecuador. Revista Tecnologica ESPOL, 20(20), 200. Retrieved from https://www.dspace.espol.edu.ec/bitstream/123456789/8387/1/Implementaci\%C 3\%B3n\%20del\%20Sistema\%20de\%20Negociaci\%C3\%B3n\%20Burs\%C3\%A1t il $\% 20 \mathrm{de} \% 20$ cheques.pdf

Prado, E. G. (2015). Proyecto y viabilidad del negocio o microempresa . España: Paraninfo.com.

Puerto, P. (2010). Globalization and entrepreneurial growth through internationalization strategies. Revista Cientifica Pensamiento y Gestión, 28, 247-248.

Robbins, S., \& Couter, M. (2014). Administración. México: Pearson.

Salazar, B. (2016). Ingeniería Industrial. Retrieved from Ingeniería Industrial online: www.ingenieriaindustrialonline.com 
Sanchez, I. (2013, 12 7). slideshare.net. Retrieved 08 09, 2017, from https://es.slideshare.net/mickienet/base-de-datos-sistema-modelo-de-gestion-dedatos

Sanchez, R. G.-M. (2015). Intervencion en la atencion sociosanitaria en instituciones. Paraninfo.com.

Secretaría Nacional de Planificación y Desarrollo. (2015). Agenda Zonal: Zona 3- Centro. Ecuador: Senplades.

Silva, A. (2013, Mayo 25). Acerca de nosotros: allanucatse.files.wordpress.com. Retrieved from allanucatse.files.wordpress.com Web site: https://allanucatse.files.wordpress.com/2011/01/tipo-de-muestreo.pdf

SISGECOM. (2011, noviembre 2). Sistemas para la gestión de la información y las comunicaciones estratégicas. Retrieved from Los indicadores de gestión como elemento de medición en las estratégias de comunicaciones y marketing: https://sisgecom.com/2011/11/02/los-indicadores-de-gestion-como-elemento-demedicion-en-las-estrategias-de-comunicaciones-y-marketing/

Smith, S. (2015). Ending Global Poverty: A Guide to What Works. macmillan.com.

Tandon. (2015). Endangered heritage: emergency evacuation of heritage collections . UNESCO Publishing.com.

Taquía, J. (2016, Mayo 22). Retail marketing para desarrollar mercados. Redalic(33), 133-155. Retrieved from http://www.redalyc.org/articulo.oa?id=337443854006

Welch, F., \& Comer, M. (2015). Alfa de Cronbach y consistencia interna de los ítems de un instrumento de medida. Estadistica Moderna, 22. Retrieved from http://www.uv.es/ friasnav/AlfaCronbach.pdf

Wigodski, J. (2014, Mayo 02). Acerca de nosotros: metodologiaeninvestigacion.blogspot.com. Retrieved from metodologiaeninvestigacion.blogspot.com Web site: http://metodologiaeninvestigacion.blogspot.com/2010/07/poblacion-ymuestra.html

Wilkins, K. (2015). Communicating Gender and Advocating Accountability in Global Development. Spriger.com.

Woodsong, C. (2011, Julio 16). Qualitative Research Methods: A DATA COLLECTOR'S FIELD GUIDE. amily Healt International, 137. Retrieved from https://www.fhi360.org/sites/default/files/media/documents/Qualitative\%20Rese arch\%20Methods\%20\%20A\%20Data\%20Collector\%27s\%20Field\%20Guide.pdf

Yousem, D. (2015). Non-Interpretive Skill for Radiology: Case Review E-Book. Elsevier Health Sciences.com.

Yrusta, L., \& Nardi, A. (2015, Julio 24). Oficina de Conocimiento Abierto: un modelo para institucionalizar el acceso abierto en las universidades. El profesional de la información, 21(6), 69-163. Retrieved from http://www.elprofesionaldelainformacion.com/contenidos/2012/noviembre/11.ht $\mathrm{ml}$ 
Zevallos, L., Pastor, R., \& Moscoso, B. (2016, Julio 17). OFERTA Y DEMANDA DE SSISTEMAS DE INFORMACIÓN EN LOS ESTABLECIMIENTOS DE SALUD DEL MINISTERIO DE SALUD: BRECHAS A NIVEL NACIONAL, POR REGIONES Y TIPO DE ESPECIALIDAD. Peru Med, 28(2), 177-85. Retrieved from www.rpmesp.ins.gob.pe/index.php/rpmesp/article/download/482/483

\section{Para citar el artículo indexado.}

Velasteguí E. \& Castro A. (2017). Plan de negocio para la creación de una MIPYMES para elaboración de mascarillas faciales caseras. Revista electrónica Visionario Digital 1(1), 49-62. Recuperado desde: http://www.visionariodigital.org/index.php/VISIONARIODIGITAL/article/view/40/47

\section{Liencia}

El artículo que se publica es de exclusiva responsabilidad de los autores y no necesariamente reflejan el pensamiento de la Revista Ciencia Digital.

El articulo queda en propiedad de la revista y, por tanto, su publicación parcial y/o total en otro medio tiene que ser autorizado por el director de la Revista Ciencia Digital.
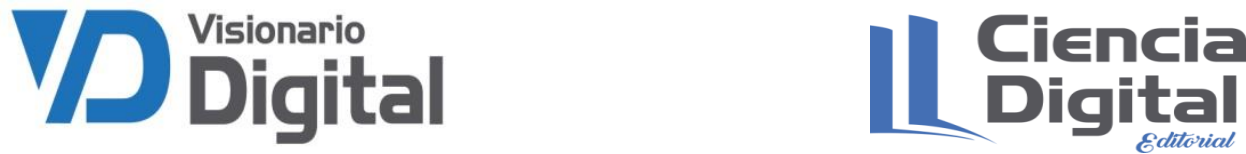